\title{
Evaluation of Changes in Depression, Anxiety, and Social Anxiety Using Smartphone Sensor Features: Longitudinal Cohort Study
}

Jonah Meyerhoff ${ }^{1}$, PhD; Tony Liu², BA; Konrad P Kording ${ }^{3,4}$, PhD; Lyle H Ungar ${ }^{2}$, PhD; Susan M Kaiser ${ }^{1}$, MPH; Chris J Karr ${ }^{5}$, MA; David C Mohr ${ }^{1}$, PhD

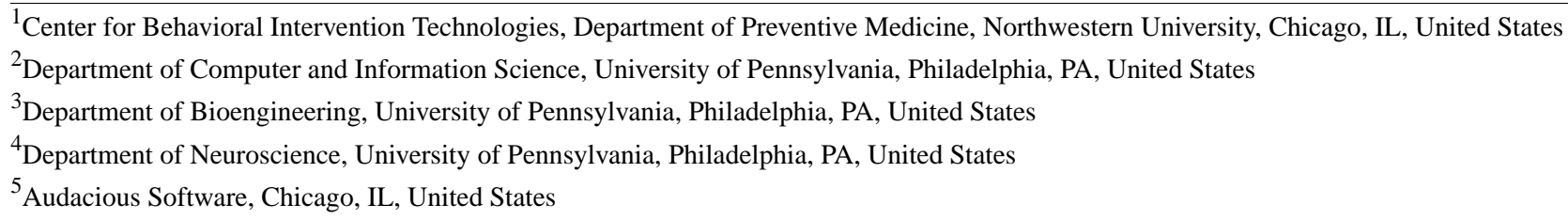

\section{Corresponding Author:}

David C Mohr, PhD

Center for Behavioral Intervention Technologies

Department of Preventive Medicine

Northwestern University

750 N Lake Shore Dr

10th Floor

Chicago, IL, 60611

United States

Phone: 13125031403

Email: d-mohr@northwestern.edu

\begin{abstract}
Background: The assessment of behaviors related to mental health typically relies on self-report data. Networked sensors embedded in smartphones can measure some behaviors objectively and continuously, with no ongoing effort.

Objective: This study aims to evaluate whether changes in phone sensor-derived behavioral features were associated with subsequent changes in mental health symptoms.

Methods: This longitudinal cohort study examined continuously collected phone sensor data and symptom severity data, collected every 3 weeks, over 16 weeks. The participants were recruited through national research registries. Primary outcomes included depression (8-item Patient Health Questionnaire), generalized anxiety (Generalized Anxiety Disorder 7-item scale), and social anxiety (Social Phobia Inventory) severity. Participants were adults who owned Android smartphones. Participants clustered into 4 groups: multiple comorbidities, depression and generalized anxiety, depression and social anxiety, and minimal symptoms.

Results: A total of 282 participants were aged 19-69 years (mean 38.9, SD 11.9 years), and the majority were female (223/282, $79.1 \%$ ) and White participants $(226 / 282,80.1 \%)$. Among the multiple comorbidities group, depression changes were preceded by changes in GPS features (Time: $r=-0.23, P=.02$; Locations: $r=-0.36, P<.001)$, exercise duration $(r=0.39 ; P=.03)$ and use of active apps $(r=-0.31 ; P<.001)$. Among the depression and anxiety groups, changes in depression were preceded by changes in GPS features for Locations ( $r=-0.20 ; P=.03)$ and Transitions $(r=-0.21 ; P=.03)$. Depression changes were not related to subsequent sensor-derived features. The minimal symptoms group showed no significant relationships. There were no associations between sensor-based features and anxiety and minimal associations between sensor-based features and social anxiety.
\end{abstract}

Conclusions: Changes in sensor-derived behavioral features are associated with subsequent depression changes, but not vice versa, suggesting a directional relationship in which changes in sensed behaviors are associated with subsequent changes in symptoms.

(J Med Internet Res 2021;23(9):e22844) doi: $\underline{10.2196 / 22844}$ 


\section{KEYWORDS}

mHealth; personal sensing; digital phenotyping; passive sensing; ecological momentary assessment; depression; anxiety; digital biomarkers; digital phenotyping; mental health assessment; mobile device; mobile phone; internet technology; psychiatric disorders; mobile phone

\section{Introduction}

\section{Background}

Behaviors such as levels of activity and social engagement are associated with common mental health conditions such as depression and anxiety [1-4]. Retrospective evaluations of these behaviors through self-report measures or interviews rely heavily on retrospective recall, which is subject to systematic biases $[5,6]$. Even more accurate methods, such as ecological momentary assessment (EMA), which acquire self-reported experiences in the course of peoples' lives [7,8], have not proven practical over extended periods outside of research settings [7]. Accelerometry data from wearable devices that measure activity levels have also been associated with depression [9], but many people stop wearing the devices within the first weeks [10].

Smartphones are becoming ubiquitous. As of $2019,81 \%$ of Americans owned a smartphone [11], as did $76 \%$ of people in countries with advanced economies, and $45 \%$ in emerging nations [12]. Smartphones are fully integrated into our lives, supporting a growing number of activities. Smartphones contain embedded networked sensors that provide continuous, objective data without user effort, which can be used to produce behavioral markers. A growing body of research suggests that these sensor data can be associated with common mental health problems [13]. Location features derived from GPS actual measurement of patterns of locations visited, time in locations, and travel in phone communications such as text messaging and phone and app use have been associated with depression, anxiety, and social anxiety [14-17].

The potential for personal mobile sensing to improve our understanding of the relationship between behavior and mental health, as well as to advance clinical care, has been widely recognized [18]. However, although there is promise, research to date has had a number of weaknesses. Many of these studies have been conducted in small, relatively homogenous groups, such as students [15,16,19-21]. Although there has been some specificity, with location features tending to identify depression $[14,15,19,22,23]$ and communication features tending to predict social anxiety [17], there have also been a few studies that have found the opposite $[16,24]$. This may be because of the heterogeneity in symptoms and comorbidities [25], which are common and have not been considered in existing research [13].

To date, studies have focused on using sensed behavioral features to estimate a person's state, either the presence or absence of a condition or symptom severity. With some exceptions, they have generally not evaluated the capacity for sensed behavioral features to predict whether symptoms will increase or decrease in the future. Among the few studies that have examined the capacity of sensed features to predict symptom change, one small study of 18 patients with bipolar disorder found that greater inconsistencies in rates of typing on a smartphone keyboard were related to future greater depressive symptom severity [26]. Relative to studies that use sensed behavior to estimate a person's state, the temporal relationship between sensed behaviors and symptom change has received relatively little attention.

\section{Objectives}

In this study, we examine the temporal relationship between changes in sensor features and subsequent changes in mental health symptoms in a large sample of participants. The aim of this exploratory study is to evaluate whether changes in classes of smartphone sensor features were associated with changes in symptom severity for depression, anxiety, and social anxiety, across all participants as well as within groups clustered based on symptoms.

\section{Methods}

\section{Participants}

Participants were recruited from July 15 to July 26, 2019, through ResearchMatch, a National Institute of Health-funded volunteer network, and the Center for Behavioral Intervention Technologies research registry. Participants were included if they were US citizens and residents, age $\geq 18$ years, could read English, and had an Android smartphone. Participants were excluded if they endorsed, via self-report, having been diagnosed with a severe mental illness, defined as bipolar disorder, schizophrenia, or other psychotic disorder. Participants were compensated for completing measures at set assessment points as well as for completing EMA check-ins. Compensation for completing assessments increased as the study period progressed, such that participants were compensated relatively less for early assessment points and relatively more for assessments toward the end of the study. No single assessment was compensated at more than US $\$ 32.50$ per assessment time point. Recruitment was advertised as a study on depression and deliberately oversampled depressed participants such that at least $50 \%$ of the sample experienced at least moderate depression symptom severity according to the 8-item Patient Health Questionnaire (PHQ-8).

\section{Procedures}

Participants downloaded the Passive Data Kit [27] mobile app, which unobtrusively collects phone sensor data and administers surveys. Web-based questionnaires were administered every 3 weeks. Participants were enrolled in the study for 16 weeks. All procedures were approved by the Northwestern University Institutional Review Board, and informed consent was obtained from all participants before participation.

\section{Measures}

Participants completed web-based symptom severity assessments at baseline and every 3 weeks until the end of the study period (ie, weeks 4, 7, 10, 13, and 16). Symptom measures included depression severity (PHQ-8) [28], generalized anxiety disorder 
(Generalized Anxiety Disorder 7-item scale [GAD-7]) [29], and social anxiety disorder (Social Phobia Inventory [SPIN]) [30]. The PHQ-8 was administered as an EMA survey after the baseline assessment point and, subsequently, had one additional assessment point (week 1) relative to other symptom measures.

Phone sensor data included GPS coordinates sampled once every 5 minutes, communication information (ie, phone logs and duration, text message logs, and length), and open apps. Assessment weeks occurred every 3 weeks, during which participants were asked each evening to label the semantic location (type of location) that they had visited for more than 10 minutes [31]. A series of maps identifying each location were presented, and participants selected the category of each place (eg, home, work, errand, entertainment, place of worship, etc).

\section{Data Analyses}

\section{Phone Sensor Feature Transformation}

\section{Overview}

We considered four categories of phone features for our analysis: GPS-derived movement and location information, semantic locations, app use, and phone-based communication (calls and texts).

To increase interpretability and reduce the number of sensor features, we aggregated features first based on their phone sensor source, as different sensor sets provide unique information. Within each sensor set, we used unit weightings that maximized the interpretability for each feature aggregation. Where possible, we used existing theory to guide our unit aggregations. All sensor features were standardized (mean-centered with unit variance) across the full sample and averaged to produce sensor groupings within the four sensor categories. A full list of features, feature calculations, and their groupings can be found in Multimedia Appendix 1.

\section{GPS-Derived Location and Movement}

Following the methodology of Saeb et al [20], we computed high-level features from GPS data that measure participant movement, including location variance (variability in GPS location), total unique location clusters, location entropy (variability in time spent at location clusters), normalized entropy, total distance traveled, average velocity, and circadian movement (extent to which sequence of locations followed a 24-hour pattern). The features were aggregated into Locations (location cluster and location variance; represents the number and variability in locations visited), Time (total entropy, normalized entropy, and circadian movement; represents the variability in time spent across locations), and Transitions (distance traveled and velocity; represents travel between locations).

\section{Semantic Location}

Labels for semantic location categories included home duration, work duration, shopping duration, social activities duration (eg, friends' homes and entertainment), religious activities duration (eg, place of worship), and exercise location duration (eg, gyms). During the nonassessment weeks, semantic labels were assigned to locations visited using GPS coordinates assigned during the assessment weeks. This allowed us to estimate the daily duration of time participants spent in each semantic location category.

\section{Communication}

The number of incoming and outgoing calls and texts, call duration, and text message length were summed to obtain daily aggregates. The feature groups were Calls and Text Messages.

\section{App Use}

Apps running in the foreground of the phone were sampled every 5 minutes. We aggregated to produce daily app use duration measurements. We grouped apps of interest into 3 categories based on previous theory that certain apps facilitate active use, whereas others elicit more passive use [32,33]. This theoretical underpinning resulted in 3 categories of app use that were manually constructed using unit weighting. Final categorizations included: Active Apps (eg, messaging, email, and maps), which required active engagement to complete the primary essential task of each app, Information Consumption Apps (eg, YouTube and web browsers) where the primary purpose was more passive consumption of information or entertainment and Social Apps (eg, Facebook, Instagram, and Snapchat), which were considered social media apps, and were generally considered as a unique category of apps [34].

\section{Population Clustering}

Heterogeneity in underlying symptom patterns may impede the ability to observe clinically meaningful relationships between sensor features and symptom severity $[13,35]$. We used a data-driven approach, performing k-means clustering on the baseline PHQ-8, GAD-7, and SPIN items [36]. We chose $\mathrm{k}=4$ using the elbow heuristic to choose the number of clusters (Multimedia Appendix 2). Qualitative analysis of these clusters showed that the 4 groups roughly corresponded to (1) a Minimal Symptom cluster $(\mathrm{n}=88)$, comprising participants characterized by low mean scores on all outcome measures; (2) a Depression andSocial Anxiety cluster $(\mathrm{n}=71)$ that included participants with predominantly moderate severity scores on the PHQ-8 and the SPIN, but low scores on the GAD-7 measures; (3) a Depression and Anxiety cluster $(\mathrm{n}=69)$, characterized by generally moderate-severe symptoms on the PHQ-8, moderate symptoms on the GAD-7, but mild ratings on the SPIN; and a (4) Multiple Comorbidities cluster $(n=54)$ characterized by elevated ratings across all three symptom measures, with a substantial proportion scoring in the severe range.

\section{Statistical Methods: Correlation of Changes in Sensors to Changes in Symptom Severity}

Figure 1 shows the strategy we used to lag, by 2 weeks, repeated measure correlations [37] of the changes in phone sensor features with changes in symptom severity. The 2-week window for sensor features, consistent with previous research [14,20], was used to allow for sufficient quality of sensor readings to match the retrospective time spans of self-report questionnaires and to maximize data available for analysis while preventing overlapping data sources (ie, symptom outcomes and concurrent sensed behavioral data) from being used at different time points. For the PHQ-8, we had six check-ins across the entire study, yielding five pairs of changes for each participant, whereas for GAD-7 and SPIN, we had five check-ins, yielding four pairs of 
changes for each participant. For analyses, in which changes in sensor features were used to estimate subsequent symptom severity, $\mathrm{Sn}_{2}-\mathrm{Sn}_{1}$ was correlated with $\mathrm{Sx}_{2}-\mathrm{Sx}_{1}$. For analyses in which changes in symptom severity were used to estimate changes in subsequent sensor features, $\mathrm{Sx}_{2}-\mathrm{Sx}_{1}$ was correlated with $\mathrm{Sn}_{2}{ }_{2}-\mathrm{Sn}_{1}{ }_{1}$. To correct for multiple comparisons, we computed adjusted $P$ values using the Benjamini-Hochberg procedure to control the false discovery rate [38].
If one assessment check-in was missing from a given pair of check-in dates, we used a within-person mean-fill method for the missing assessment. Any pair of assessment check-ins that had missing phone sensor data was discarded from analyses. Power calculations revealed that a sample size of 255 would be required to detect an effect size (correlation, $|\rho|$ ) of 0.2 at an $\alpha$ of .05 and power $(\beta)$ of .90 .

Figure 1. The sensor window preceding (a) and proceeding (b) the assessment check-ins. Correlations are run as corrected $\left(\mathrm{Sx}_{2}-\mathrm{Sx}_{1}, \mathrm{Sn}_{2}-\mathrm{Sn}_{1}\right)$ and corrected $\left(\mathrm{Sx}_{2}-\mathrm{Sx}_{1}, \mathrm{Sn}_{2}-\mathrm{Sn}_{1}\right)$.

\section{a. Change in sensors predicting change in symptom measure}

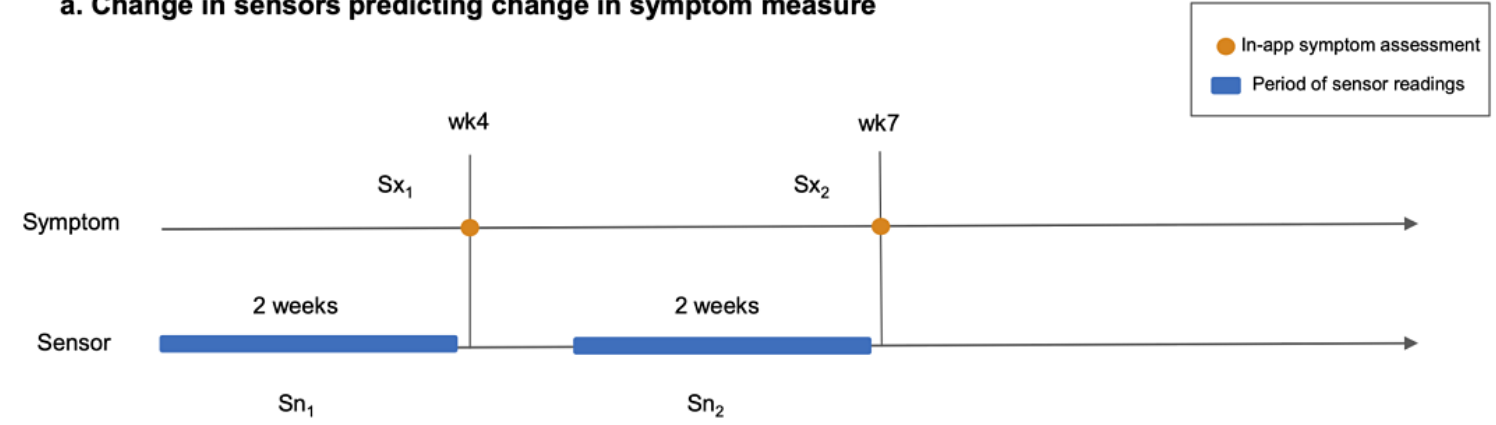

b. Change in symptom measures predicting change in sensors

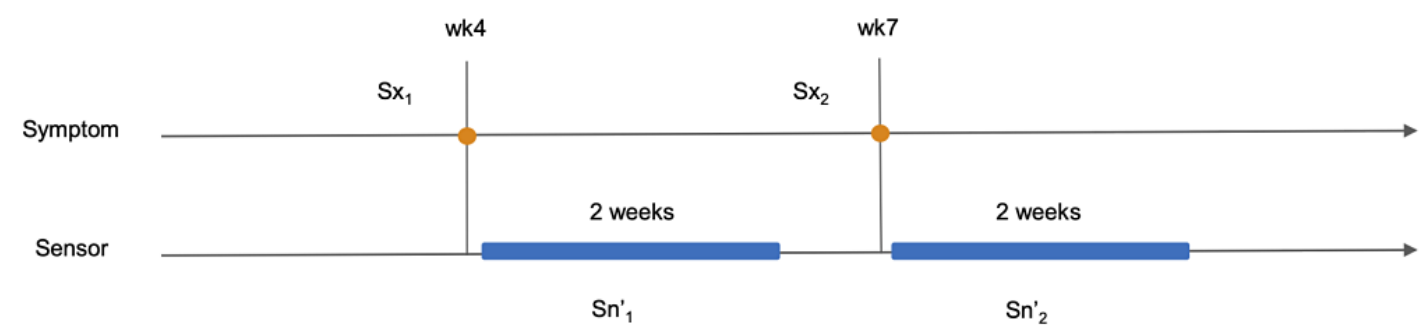

\section{Results}

\section{Participants}

The flow of participants in this study is shown in Figure 2.

Participant demographic and baseline symptom severity characteristics across the entire sample and participant clusters are detailed in Multimedia Appendix 3. 
Figure 2. Participant flow diagram. GAD-7: Generalized Anxiety Disorder 7-item scale; PHQ-8: 8-item Patient Health Questionnaire; SPIN: Social Phobia Inventory.

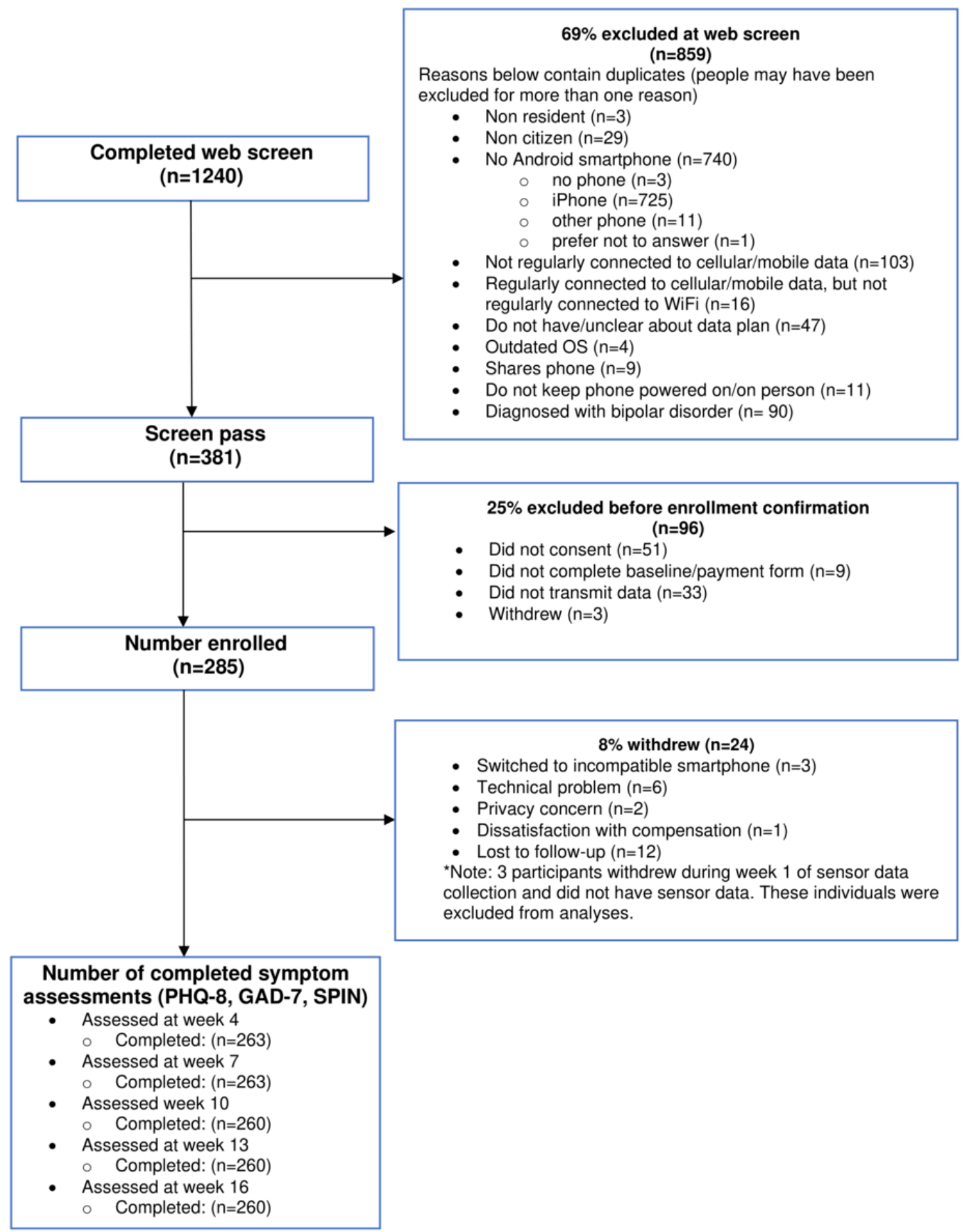

\section{Symptom Change Over Time}

Ordinary least square regression revealed no significant change in symptom severity as a function of time (PHQ-8: $P=.80$; GAD-7: $P=.83$; SPIN: $P=.57)$. However, there was substantial within-participant variability depending on the symptom measure, with mean SDs of 2.66, 3.50, and 5.90, for the PHQ-8, GAD-7, and SPIN, respectively.

\section{Association Between Sensor-Derived Behavioral Feature Changes and Subsequent Symptom Severity Change}

\section{Overview}

Table 1 displays the repeated measure correlations primary outcomes by symptom cluster. 
Table 1. Repeated measure correlations between sensor and symptom changes and symptom and sensor changes ${ }^{\mathrm{a}}$.

\begin{tabular}{|c|c|c|c|c|c|c|c|c|c|c|}
\hline \multirow[t]{3}{*}{ Characteristics } & \multicolumn{10}{|c|}{ Repeated measure correlations } \\
\hline & \multicolumn{5}{|c|}{$\begin{array}{l}\text { Change in sensor features association with change in } \\
\text { symptom measure }\end{array}$} & \multicolumn{5}{|c|}{$\begin{array}{l}\text { Change in symptom measure association with change in } \\
\text { sensor features }\end{array}$} \\
\hline & Value, $\mathrm{n}$ & $\operatorname{dof}^{b}$ & $\mathrm{r}_{\mathrm{rm}}^{\mathrm{c}}$ & $\begin{array}{l}P \text { value } \\
\text { (uncorrected) }\end{array}$ & $\begin{array}{l}P \text { value } \\
(\text { corrected })^{\mathrm{d}}\end{array}$ & Value, $n$ & $\operatorname{dof}^{b}$ & $r_{r m}$ & $\begin{array}{l}P \text { value } \\
\text { (uncorrected) }\end{array}$ & $\begin{array}{l}P \text { value } \\
(\text { corrected })^{\mathrm{d}}\end{array}$ \\
\hline
\end{tabular}

Symptom measure: PHQ-8

\section{Full sample}

\section{GPS features}

Locations

Time

223

80

$802-0.12<.001$

Transitions

223

$802-0.12<.001$

Semantic location

Home $-225$

$\begin{array}{lll}806 & 0.054 & .13\end{array}$

duration

Work duration

192

$\begin{array}{lll}700 \quad 0.026 & .50\end{array}$

Shopping

$\begin{array}{llll}212 & 767 & 0.009 & .80\end{array}$

duration

Social activities

$\begin{array}{llll}219 & 790 & -0.062 & .08\end{array}$

duration

Religious activities 54

duration

Exercise location $\quad 85$

duration

\section{Communication}

SMS text

$\begin{array}{llll}223 & 796 & -0.034 & .34\end{array}$

messages

Calls

225

80

$\begin{array}{lll}195 & -0.084 & .24\end{array}$

$\begin{array}{lll}314 & 0.18 \quad .001\end{array}$

App use

$\begin{array}{lcccc}\text { Active apps } & 226 & 809 & -0.041 & .24 \\ \begin{array}{l}\text { Information con- } \\ \text { sumption apps }\end{array} & 225 & 805 & 0.026 & .47 \\ \text { Social apps } & 208 & 748 & 0.073 & .05\end{array}$

Subgroups (features with corrected $P \geq .1$ omitted)

\section{Multiple comorbidities}

Time

41

$143 \quad-0.36<.001$

$\begin{array}{lll}143 & -0.23 & .005\end{array}$

Transitions

41

$\begin{array}{lll}143 & -0.18 \quad .03\end{array}$

Exercise location 11

$\begin{array}{lll}41 & 0.39 & .01\end{array}$

duration

Active apps

42

$146 \quad-0.31<.001$

Depression and anxiety

$\begin{array}{lllll}\text { Locations } & 56 & 204 & -0.20 & .005 \\ \text { Transitions } & 56 & 204 & -0.21 & .002\end{array}$

Depression and social anxiety
61

$\begin{array}{lll}218 & -0.17 & .01\end{array}$

$<.001$
.003
.003

225

$801<0.001 \quad 98$

.98

225

$\begin{array}{lll}801 & -0.006 & .86\end{array}$

.97

225

$\begin{array}{lll}801 \quad 0.020 & .56\end{array}$

.94

.23

224

$801 \quad 0.017 \quad .64$

.95

.53

190

$\begin{array}{lll}691 & -0.012 & .74\end{array}$

.97

.80

210

$\begin{array}{lll}210 & -0.005 & .88\end{array}$

.97

.17

207

$\begin{array}{lll}207 & -0.021 & .57\end{array}$

.94

.36

44

$\begin{array}{lll}44 & -0.14 & .08\end{array}$

.005

81

$\begin{array}{lll}81 & -0.13 \quad .02\end{array}$

.46

221

$\begin{array}{lll}790 & -0.062 & .08\end{array}$

.31

$\begin{array}{llll}221 & 786 & -0.023 & .52\end{array}$

.94

.50

.36

225

$\begin{array}{lll}807 & -0.004 & .90\end{array}$

.97

.53

226

$\begin{array}{lll}809 & -0.072 & .04\end{array}$

.30

.14

$207 \quad 746 \quad-0.021 \quad .57$

.94

$\begin{array}{llllll}<.001 & 41 & 143 & 0.021 & .80 & .93 \\ .02 & 41 & 143 & -0.061 & .46 & .70 \\ .07 & 41 & 143 & 0.051 & .55 & .74 \\ .03 & 10 & 33 & -0.13 & .45 & .70 \\ <.001 & 42 & 146 & 0.10 & .22 & .70\end{array}$




\begin{tabular}{|c|c|c|c|c|c|c|c|c|c|c|}
\hline \multirow[t]{3}{*}{ Characteristics } & \multicolumn{10}{|c|}{ Repeated measure correlations } \\
\hline & \multicolumn{5}{|c|}{$\begin{array}{l}\text { Change in sensor features association with change in } \\
\text { symptom measure }\end{array}$} & \multicolumn{5}{|c|}{$\begin{array}{l}\text { Change in symptom measure association with change in } \\
\text { sensor features }\end{array}$} \\
\hline & Value, $\mathrm{n}$ & $\operatorname{dof}^{b}$ & $r_{r m}^{c}$ & $\begin{array}{l}P \text { value } \\
\text { (uncorrected) }\end{array}$ & $\begin{array}{l}P \text { value } \\
(\text { corrected })^{\mathrm{d}}\end{array}$ & Value, $\mathrm{n}$ & $\operatorname{dof}^{\mathrm{b}}$ & $\mathrm{r}_{\mathrm{rm}}$ & $\begin{array}{l}P \text { value } \\
\text { (uncorrected) }\end{array}$ & $\begin{array}{l}P \text { value } \\
(\text { corrected })^{\mathrm{d}}\end{array}$ \\
\hline Time & 61 & 218 & -0.16 & .02 & .08 & 62 & 218 & 0.025 & .71 & .93 \\
\hline $\begin{array}{l}\text { Social Activities } \\
\text { Duration }\end{array}$ & 60 & 214 & -0.17 & .01 & .08 & 57 & 208 & 0.006 & .93 & .93 \\
\hline \multicolumn{11}{|l|}{ Symptom measure: SPIN ${ }^{\mathrm{g}}$} \\
\hline \multicolumn{11}{|c|}{ Depression and social anxiety (features with corrected $P \geq .1$ omitted) } \\
\hline Calls & 66 & 195 & 0.25 & $<.001$ & .005 & 66 & 193 & -0.045 & .53 & .73 \\
\hline
\end{tabular}

\footnotetext{
${ }^{\mathrm{a}}$ There were no significant associations between sensor features and subsequent 8-item Patient Health Questionnaire (PHQ-8) symptoms or PHQ-8 symptoms and subsequent sensor features within the minimal symptoms group. There were also no significant associations between sensor features and subsequent Generalized Anxiety Disorder 7-item scale (GAD-7) symptoms or GAD-7 symptoms and subsequent sensor features for any subgroup.

${ }^{b} \mathrm{dof}=\mathrm{n}(\mathrm{k}-1)$, where $\mathrm{n}$ is the total number of participants and $\mathrm{k}$ is the average number of observations per participant.

${ }^{\mathrm{c}}$ Repeated measures correlation coefficient.

${ }^{\mathrm{d}}$ Benjamini-Hochberg corrected $P$ values. Identical $P$ values are due to the recursive definition of the Benjamini-Hochberg correction; it is possible for corrected $P$ values to be identical, especially for nonsignificant correlations.
}

${ }^{\mathrm{e}}$ PHQ-8: Patient Health Questionnaire-8 item.

${ }^{\mathrm{f}}$ Full results are provided for Patient Health Questionnaire-8 item results in the full sample only to display all sensed behavioral features. Thereafter only relationships with corrected $P<.1$ are displayed.

${ }^{\mathrm{g}}$ SPIN: Social Phobia Inventory.

\section{Location Features}

Patterns in individuals' movements were associated with subsequent changes in psychological symptoms, specifically depressive symptoms. Changes in GPS-derived Location were negatively associated with changes in the PHQ-8 in two of three symptom clusters, Multiple Comorbidities ( $r=-0.36 ; P<.001)$, Depression and Anxiety ( $r=-.20 ; P=.03$ ), and the full sample $(r=-0.17 ; P<.001)$ and trended toward significance in the Depression and Social Anxiety cluster $(r=-0.16 ; P=.08)$, but the results were not statistically significant. Changes in GPS-derived Time were negatively associated with changes in PHQ-8 within the Multiple Comorbidities cluster $(r=-0.23$; $P=.02)$ and the full sample $(r=-0.12 ; P=.003)$ and trended toward significance in the Depression and Social Anxiety cluster ( $r=-0.16 ; P=.08$ ), but the results were not statistically significant. Changes in GPS-derived Transitions were negatively correlated with changes in PHQ-8 for the Depression and Anxiety cluster $(r=-0.21 ; P=.03)$ and the full sample $(r=-0.12$; $P=.003$ ) and trended toward significance in the Multiple Comorbidities cluster $(r=-0.18 ; P=.07)$, but the results were not statistically significant. There were no significant relationships for the Minimal Symptom cluster for the PHQ-8 and no significant relationships between GPS features and subsequent changes in the GAD-7 or SPIN.

Certain types of semantic locations were also associated with PHQ-8 changes. In the depression and social anxiety cluster, there was a trend toward significance for Social Activity Duration, which was negatively correlated with changes in the PHQ-8 ( $r=-0.17 ; P=.08)$, but the results were not statistically significant. Within the Multiple Comorbidities symptom cluster and the full sample, Exercise Location Duration was positively associated with subsequent changes in PHQ-8 scores (Multiple
Comorbidities cluster: $r=0.39, P=.03$; full sample: $r=0.18$, $P=.005)$. This unexpected association between changes in exercise locations and changes in PHQ-8 was likely due to a preponderance of individuals who spent no time in exercise-based locations (ie, zero change in Exercise Location Duration from zero time spent in exercise locations), thus overweighting the data of some individuals who saw increased time spent in exercise-based locations with increased PHQ-8. There were no significant relationships within the minimal symptom cluster for the PHQ-8, and no significant relationships were found between semantic location features and subsequent changes in the GAD-7 or SPIN.

\section{Telephone Calls}

Within the Depression and Social Anxiety baseline cluster, increases in calls were associated with increases in SPIN scores $(r=0.25 ; P<.001)$.

\section{App Use}

Changes in Active App Use were negatively correlated ( $r=-0.31$; $P<.001)$ with changes in PHQ-8 scores within the Multiple Comorbidities cluster.

\section{Association Between Changes in Symptom Severity and Subsequent Change in Sensor-Derived Behavioral Features}

There were no significant correlations between changes in any symptom severity measures and subsequent changes in sensor-derived behavioral features.

\section{Missing Data}

Across all treatment weeks, missingness (ie, the number of missing observations/total number of possible observations for 
all 282 participants) was higher for the PHQ-8 (277/1692, $16.37 \%)$ assessments than for the GAD-7 (104/1410, 7.38\%) and SPIN (104/1410, 7.38\%). PHQ-8 assessments were administered through our smartphone app, whereas the GAD-7 and SPIN were administered through REDCap (Research Electronic Data Capture) [39]. In addition, across symptom outcome measures and relative to baseline symptom levels (Multimedia Appendix 3), those with missing assessments tended to have slightly higher baseline symptom severity (PHQ-8 range: 10.94-12.82; GAD-7 range: 9.95-11.5; and SPIN range: 24.52-27.48).

\section{Discussion}

\section{Principal Findings}

Changes in numerous phone sensor-derived behavioral features were associated with subsequent changes in mental health symptoms among people with elevated symptoms of depression. However, changes in symptoms were not associated with subsequent changes in behavioral features. GPS location features were fairly consistently and negatively, albeit modestly, related to subsequent changes in depression severity across symptom groups. This is consistent with a number of previous relatively small studies showing correlations between GPS features and depression $[14,19,20,22,40,41]$. This larger study confirms these earlier findings, and importantly, indicates a directional relationship in which GPS features are associated with subsequent increases or decreases in depressive symptoms, but not with anxiety or social anxiety.

The types of locations (work, shopping, etc) people visited were less consistently related to changes in depression. This does not necessarily mean that specific locations are unimportant at the individual level: one person may like shopping, whereas another may detest it. However, this suggests that patterns of movement through geographic space, irrespective of the destinations or locations to which one travels, are indicators of symptom change among people with depression. We speculate that this may reflect a loss of motivation expressed through geographic movement. Perhaps more speculatively, it may also be that changes in neurocognitive mechanisms, such as executive control, affect, and motivation, impact both depression and basic mechanisms involved in movement through geographic space $[42,43]$.

The different constellations of symptoms that participants experience impact the salience of some sensed behaviors in predicting outcomes. For example, only within participants in the Depression and Social Anxiety symptom cluster was the amount of time spent in locations related to social activities associated with (at the trend level) subsequent change in depressive symptoms, suggesting that although locations are generally useful for depression prediction, social activities may be particularly useful when social anxiety symptoms are present. Active App Use (texting, email, and mapping) was associated with depression change among those with multiple and more severe comorbidities. Although GPS features were generally useful, features integrating time and location were not useful among those with comorbid generalized anxiety, and features measuring transitioning between locations were not useful for those with comorbid social anxiety. Thus, there was support for the notion that symptom constellations are important considerations for some features.

Increases in telephone calls were associated with increases in social anxiety symptoms among clusters characterized by depression and social anxiety. This finding notwithstanding, the capacity for sensor-derived features to be associated with changes in social anxiety symptoms was not as consistent as that for depressive symptoms. Furthermore, we did not find any associations between sensor-derived features and generalized anxiety symptom changes.

These findings indicate that sensor-derived behavioral features, which are objective and can be acquired with reduced participant effort, can be a useful tool for investigating the role of some behaviors in changing depressive symptom severity. There has been much speculation about the clinical potential of mobile sensing $[18,44]$. The effect sizes are modest, albeit consistent with many other studies that have examined the use of sensed behavioral features to estimate the presence or severity of symptoms $[16,19]$. This study does not support the use of phone sensor data alone in monitoring symptom changes in mental health populations; however, phone sensor data may be useful in conjunction with other networked sensing tools such as wearables. Phone sensor data may be useful for digital mental health interventions [45]. Just-in-time adaptive interventions $[46,47]$ use individualized data to predict risk and deliver context-aware intervention material that is adaptive. These approaches are increasingly applied in digital health interventions, such as identifying when to send messages to increase physical activity such as step counts [48]. The promise of delivering motivational messages at opportune moments that reinforce behavioral activation strategies, such as visiting someplace new, spending more time outside of the home or work, or engaging socially, has the potential to improve engagement with these tools and reduce depression.

\section{Limitations}

This study had several limitations. First, the exploratory nature of this study requires interpreting results with caution and necessitates that future work must explicitly test the a priori hypotheses arising from these results. Next, although we establish significant temporal relationships between sensed behavior changes and subsequent changes in symptom severity, our study is not experimental and does not establish causal relationships. Furthermore, our sensor feature aggregations were limited to single sensor sources and were constructed to maximize interpretability; however, future studies that use data-driven aggregations are necessary to help inform feature aggregations across sensor modalities. Although aggregating across sensor modalities presents a challenge for interpretability, future work that examines cross-sensor aggregations could yield improved estimation of sensed behaviors and, subsequently, more robust associations with changes in symptoms. In addition, although these findings provide some support for the hypothesis that sensed behavior change is associated with subsequent changes in depression and not vice versa, this study examined the associations between changes in sensor features and subsequent changes in symptom severity measures lagged by 
2 weeks, and therefore should not be generalized to periods beyond the 2-week window. Another limitation is that our sample only included those who used Android devices and agreed to participate in this research. App use and communication data are not readily available for iOS devices. Regarding data missingness, across all the surveys, individuals who had missing data had higher baseline symptom severity than the overall sample, though not dramatically so; thus, data were missing not at random. These missingness rates are in line with established criteria that are often used as the standard for good trial data [49]. Finally, although we controlled for multiple analyses, we nonetheless caution against overinterpretation of more isolated findings that need to be replicated in future studies.

\section{Conclusions}

The ubiquity of smartphones with networked sensors has opened up new opportunities to identify behavioral markers related to mental health that can be acquired continuously and effortlessly. Changes in movement through geographic space were consistently associated with subsequent changes in depressive symptoms; however, there was no evidence that changes in depression were associated with subsequent changes in sensed behaviors. This supports a directional relationship in which changes in movement patterns precede symptom change, but symptom change does not precede changes in movement behaviors.

\section{Acknowledgments}

This work was supported by a grant from the National Institute of Mental Health (5R01MH111610) to DCM and KPK. DCM had full access to all the data in the study and takes responsibility for the integrity of the data and the accuracy of the data analysis. JM is supported by a grant from the National Institute of Mental Health (T32MH115882). The content is solely the responsibility of the authors and does not necessarily represent the official views of the National Institutes of Health.

\section{Conflicts of Interest}

DCM has accepted consulting fees from Apple Inc, Pear Therapeutics, Otsuka Pharmaceuticals, and the One Mind Foundation. He also accepted royalties from Oxford Press and has an ownership interest in Adaptive Health, Inc. SMK accepted the consulting fees from Adaptive Health, Inc. CJK is the founder and sole developer at Audacious Software, LLC, which provides academic and research software development to a variety of academic, nonprofit, and commercial entities that are similar to the contributions to this work. None of the other authors have any competing interests to declare.

\section{Multimedia Appendix 1}

Sensor features and groupings.

[PDF File (Adobe PDF File), 139 KB-Multimedia Appendix 1]

\section{Multimedia Appendix 2}

Symptom cluster elbow plot. [PDF File (Adobe PDF File), 132 KB-Multimedia Appendix 2]

\section{Multimedia Appendix 3}

Demographics and baseline characteristics. [PDF File (Adobe PDF File), 135 KB-Multimedia Appendix 3]

\section{References}

1. Cacioppo JT, Hawkley LC, Thisted RA. Perceived social isolation makes me sad: 5-year cross-lagged analyses of loneliness and depressive symptomatology in the Chicago Health, Aging, and Social Relations Study. Psychol Aging 2010 Jun;25(2):453-463 [FREE Full text] [doi: 10.1037/a0017216] [Medline: 20545429]

2. Hiles SA, Lamers F, Milaneschi Y, Penninx BW. Sit, step, sweat: longitudinal associations between physical activity patterns, anxiety and depression. Psychol Med 2017 Jun;47(8):1466-1477. [doi: 10.1017/S0033291716003548] [Medline: 28137333]

3. Pereira SM, Geoffroy M, Power C. Depressive symptoms and physical activity during 3 decades in adult life: bidirectional associations in a prospective cohort study. JAMA Psychiatry 2014 Dec 01;71(12):1373-1380. [doi: 10.1001/jamapsychiatry.2014.1240] [Medline: 25321867]

4. Da Silva MA, Singh-Manoux A, Brunner EJ, Kaffashian S, Shipley MJ, Kivimäki M, et al. Bidirectional association between physical activity and symptoms of anxiety and depression: the Whitehall II study. Eur J Epidemiol 2012 Jul;27(7):537-546 [FREE Full text] [doi: 10.1007/s10654-012-9692-8] [Medline: 22623145]

5. Tversky A, Kahneman D. Availability: A heuristic for judging frequency and probability. Cognit Psychol 1973 Sep;5(2):207-232. [doi: 10.1016/0010-0285(73)90033-9] 
6. Shiffman S, Stone AA, Hufford MR. Ecological momentary assessment. Annu Rev Clin Psychol 2008;4:1-32. [doi: 10.1146/annurev.clinpsy.3.022806.091415] [Medline: 18509902]

7. Csikszentmihalyi M. In: de Vries MW, editor. The Experience of Psychopathology: Investigating Mental Disorders in their Natural Settings. Cambridge: Cambridge University Press; 1992.

8. Killingsworth MA, Gilbert DT. A wandering mind is an unhappy mind. Science 2010 Nov 12;330(6006):932 [FREE Full text] [doi: 10.1126/science.1192439] [Medline: 21071660]

9. Cruz BD, Alfonso-Rosa RM, McGregor D, Chastin SF, Palarea-Albaladejo J, Cruz JD. Sedentary behaviour is associated with depression symptoms: Compositional data analysis from a representative sample of 3233 US adults and older adults assessed with accelerometers. J Affect Disord 2020 Mar 15;265:59-62. [doi: 10.1016/j.jad.2020.01.023] [Medline: 31959584]

10. Lee H, Lee Y. A look at wearable abandonment. In: Proceedings of the 18th IEEE International Conference on Mobile Data Management (MDM). 2017 Presented at: 18th IEEE International Conference on Mobile Data Management (MDM); May 29-June 1, 2017; Daejeon, Korea (South) p. 392-393. [doi: 10.1109/mdm.2017.70]

11. Demographics of mobile device ownership and adoption in the United States. Pew Research Center. 2021. URL: https:/ /www.pewresearch.org/internet/fact-sheet/mobile/ [accessed 2021-08-10]

12. Silver L. Smartphone ownership is growing rapidly around the world, but not always equally. Pew Research Center. 2019 Feb. URL: https://www.pewresearch.org/global/2019/02/05/smartphone-ownership-is-growing-rapidly-around-theworld-but-not-always-equally/ [accessed 2021-08-10]

13. Mohr DC, Zhang M, Schueller SM. Personal Sensing: Understanding mental health using ubiquitous sensors and machine learning. Annu Rev Clin Psychol 2017 May 08;13:23-47. [doi: 10.1146/annurev-clinpsy-032816-044949] [Medline: 28375728]

14. Saeb S, Zhang M, Karr CJ, Schueller SM, Corden ME, Kording KP, et al. Mobile phone sensor correlates of depressive symptom severity in daily-life behavior: an exploratory study. J Med Internet Res 2015 Jul 15;17(7):e175 [FREE Full text] [doi: 10.2196/jmir.4273] [Medline: 26180009]

15. Ware S, Yue C, Morillo R, Lu J, Shang C, Bi J, et al. Predicting depressive symptoms using smartphone data. Smart Health 2020 Mar;15:100093. [doi: 10.1016/j.smhl.2019.100093]

16. Chow PI, Fua K, Huang Y, Bonelli W, Xiong H, Barnes LE, et al. Using mobile sensing to test clinical models of depression, social anxiety, state affect, and social isolation among college students. J Med Internet Res 2017 Mar 03;19(3):e62 [FREE Full text] [doi: 10.2196/jmir.6820] [Medline: 28258049]

17. Gao Y, Li A, Zhu T, Liu X, Liu X. How smartphone usage correlates with social anxiety and loneliness. PeerJ 2016;4:e2197 [FREE Full text] [doi: 10.7717/peerj.2197] [Medline: 27478700]

18. Insel TR. Digital Phenotyping: Technology for a new science of behavior. J Am Med Assoc 2017 Oct 03;318(13):1215-1216. [doi: 10.1001/jama.2017.11295] [Medline: 28973224]

19. Farhan A, Yue C, Morillo R, Ware S, Lu J, Bi J, et al. Behavior vs. introspection: refining prediction of clinical depression via smartphone sensing data. In: Proceedings of the IEEE Wireless Health (WH). 2016 Presented at: IEEE Wireless Health (WH); Oct. 25-27, 2016; Bethesda, MD, USA. [doi: 10.1109/wh.2016.7764553]

20. Saeb S, Lattie EG, Schueller SM, Kording KP, Mohr DC. The relationship between mobile phone location sensor data and depressive symptom severity. PeerJ 2016;4:e2537 [FREE Full text] [doi: 10.7717/peerj.2537] [Medline: 28344895]

21. Seppälä J, De Vita I, Jämsä T, Miettunen J, Isohanni M, Rubinstein K, M-RESIST Group, et al. Mobile phone and wearable sensor-based mHealth approaches for psychiatric disorders and symptoms: systematic review. JMIR Ment Health 2019 Feb 20;6(2):e9819 [FREE Full text] [doi: 10.2196/mental.9819] [Medline: 30785404]

22. Palmius N, Tsanas A, Saunders KE, Bilderbeck AC, Geddes JR, Goodwin GM, et al. Detecting bipolar depression from geographic location data. IEEE Trans Biomed Eng 2017 Dec;64(8):1761-1771 [FREE Full text] [doi: 10.1109/TBME.2016.2611862] [Medline: 28113247]

23. Canzian L, Musolesi M. Trajectories of depression: unobtrusive monitoring of depressive states by means of smartphone mobility traces analysis. In: Proceedings of the 2015 ACM International Joint Conference on Pervasive and Ubiquitous Computing. 2015 Presented at: UbiComp '15: The 2015 ACM International Joint Conference on Pervasive and Ubiquitous Computing; September 7 - 11, 2015; Osaka Japan p. 1293-1304. [doi: 10.1145/2750858.2805845]

24. Razavi R, Gharipour A, Gharipour M. Depression screening using mobile phone usage metadata: a machine learning approach. J Am Med Inform Assoc 2020 Apr 01;27(4):522-530. [doi: 10.1093/jamia/ocz221] [Medline: 31977041]

25. Vittengl JR, Clark LA, Smits JA, Thase ME, Jarrett RB. Do comorbid social and other anxiety disorders predict outcomes during and after cognitive therapy for depression? J Affect Disord 2019 Jan 01;242:150-158 [FREE Full text] [doi: 10.1016/j.jad.2018.08.053] [Medline: 30176494]

26. Stange JP, Zulueta J, Langenecker SA, Ryan KA, Piscitello A, Duffecy J, et al. Let your fingers do the talking: Passive typing instability predicts future mood outcomes. Bipolar Disord 2018 May 08;20(3):285-288 [FREE Full text] [doi: 10.1111/bdi.12637] [Medline: 29516666]

27. Passive data kit. Audacious Software. 2018. URL: https://passivedatakit.org/ [accessed 2021-08-10]

28. Kroenke K, Strine TW, Spitzer RL, Williams JB, Berry JT, Mokdad AH. The PHQ-8 as a measure of current depression in the general population. J Affect Disord 2009 Apr;114(1-3):163-173. [doi: 10.1016/j.jad.2008.06.026] [Medline: 18752852] 
29. Spitzer RL, Kroenke K, Williams JB, Löwe B. A brief measure for assessing generalized anxiety disorder: the GAD-7. Arch Intern Med 2006 May 22;166(10):1092-1097. [doi: 10.1001/archinte.166.10.1092] [Medline: 16717171]

30. Connor KM, Davidson JR, Churchill LE, Sherwood A, Foa E, Weisler RH. Psychometric properties of the Social Phobia Inventory (SPIN). New self-rating scale. Br J Psychiatry 2000 Apr 02;176(4):379-386. [doi: 10.1192/bjp.176.4.379] [Medline: 10827888 ]

31. Saeb S, Lattie EG, Kording KP, Mohr DC. Mobile phone detection of semantic location and its relationship to depression and anxiety. JMIR Mhealth Uhealth 2017 Aug 10;5(8):e112 [FREE Full text] [doi: 10.2196/mhealth.7297] [Medline: 28798010]

32. Montague E, Xu J. Understanding active and passive users: the effects of an active user using normal, hard and unreliable technologies on user assessment of trust in technology and co-user. Appl Ergon 2012 Jul;43(4):702-712 [FREE Full text] [doi: 10.1016/j.apergo.2011.11.002] [Medline: 22192788]

33. Escobar-Viera CG, Shensa A, Bowman ND, Sidani JE, Knight J, James AE, et al. Passive and active social media use and depressive symptoms among United States adults. Cyberpsychol Behav Soc Netw 2018 Jul;21(7):437-443. [doi: 10.1089/cyber.2017.0668] [Medline: 29995530]

34. Harari GM, Müller SR, Stachl C, Wang R, Wang W, Bühner M, et al. Sensing sociability: Individual differences in young adults' conversation, calling, texting, and app use behaviors in daily life. J Pers Soc Psychol 2020 Jul;119(1):204-228. [doi: 10.1037/pspp0000245] [Medline: 31107054]

35. Chekroud AM, Gueorguieva R, Krumholz HM, Trivedi MH, Krystal JH, McCarthy G. Reevaluating the efficacy and predictability of antidepressant treatments: a symptom clustering approach. JAMA Psychiatry 2017 Apr 01;74(4):370-378 [FREE Full text] [doi: 10.1001/jamapsychiatry.2017.0025] [Medline: 28241180]

36. Taylor S, Jaques N, Nosakhare E, Sano A, Picard R. Personalized multitask learning for predicting tomorrow's mood, stress, and health. IEEE Trans Affect Comput 2020;11(2):200-213. [doi: 10.1109/TAFFC.2017.2784832] [Medline: 32489521]

37. Bakdash JZ, Marusich LR. Repeated measures correlation. Front Psychol 2017 Apr 7;8:00456. [doi: 10.3389/fpsyg.2017.00456]

38. Benjamini Y, Hochberg Y. Controlling the false discovery rate: a practical and powerful approach to multiple testing. J Royal Stat Soc Series B (Methodol) 2018 Dec 05;57(1):289-300. [doi: 10.1111/j.2517-6161.1995.tb02031.x]

39. Harris PA, Taylor R, Thielke R, Payne J, Gonzalez N, Conde JG. Research electronic data capture (REDCap)--a metadata-driven methodology and workflow process for providing translational research informatics support. J Biomed Inform 2009 Apr;42(2):377-381 [FRE Full text] [doi: 10.1016/j.jbi.2008.08.010] [Medline: 18929686]

40. Pratap A, Atkins DC, Renn BN, Tanana MJ, Mooney SD, Anguera JA, et al. The accuracy of passive phone sensors in predicting daily mood. Depress Anxiety 2019 Jan 21;36(1):72-81. [doi: 10.1002/da.22822] [Medline: 30129691]

41. Ben-Zeev D, Scherer EA, Wang R, Xie H, Campbell AT. Next-generation psychiatric assessment: Using smartphone sensors to monitor behavior and mental health. Psychiatr Rehabil J 2015 Sep;38(3):218-226 [FREE Full text] [doi: 10.1037/prj0000130] [Medline: 25844912]

42. Pykett J. Geography and neuroscience: Critical engagements with geography's “neural turn”. Trans Inst Br Geogr 2017 Oct 06;43(2):154-169. [doi: 10.1111/tran.12213]

43. Clark L, Chamberlain SR, Sahakian BJ. Neurocognitive mechanisms in depression: implications for treatment. Annu Rev Neurosci 2009;32:57-74. [doi: 10.1146/annurev.neuro.31.060407.125618] [Medline: 19400725]

44. Torous J, Staples P, Onnela J. Realizing the potential of mobile mental health: new methods for new data in psychiatry. Curr Psychiatry Rep 2015 Aug;17(8):602. [doi: 10.1007/s11920-015-0602-0] [Medline: 26073363]

45. Huckvale K, Venkatesh S, Christensen H. Toward clinical digital phenotyping: a timely opportunity to consider purpose, quality, and safety. NPJ Digit Med 2019;2:88 [FREE Full text] [doi: 10.1038/s41746-019-0166-1] [Medline: $\underline{31508498]}$

46. Klasnja P, Hekler EB, Shiffman S, Boruvka A, Almirall D, Tewari A, et al. Microrandomized trials: An experimental design for developing just-in-time adaptive interventions. Health Psychol 2015 Dec;34 Suppl:1220-1228. [doi: 10.1037/hea0000305] [Medline: 26651463]

47. Nahum-Shani I, Smith SN, Spring BJ, Collins LM, Witkiewitz K, Tewari A, et al. Just-in-Time Adaptive Interventions (JITAIs) in Mobile Health: Key Components and Design Principles for Ongoing Health Behavior Support. Ann Behav Med 2016 Sep 23. [doi: 10.1007/s12160-016-9830-8] [Medline: 27663578]

48. Liao P, Greenewald K, Klasnja P, Murphy S. Personalized HeartSteps: A reinforcement learning algorithm for optimizing physical activity. ArXiv: Computer Science - Machine Learning. 2019. URL: http://arxiv.org/abs/1909.03539 [accessed 2020-03-03]

49. Maher CG, Sherrington C, Herbert RD, Moseley AM, Elkins M. Reliability of the PEDro scale for rating quality of randomized controlled trials. Phys Ther 2003 Aug;83(8):713-721. [Medline: 12882612]

\section{Abbreviations}

EMA: ecological momentary assessment

GAD-7: Generalized Anxiety Disorder 7-item scale

PHQ-8: 8-item Patient Health Questionnaire 
REDCap: Research Electronic Data Capture

SPIN: Social Phobia Inventory

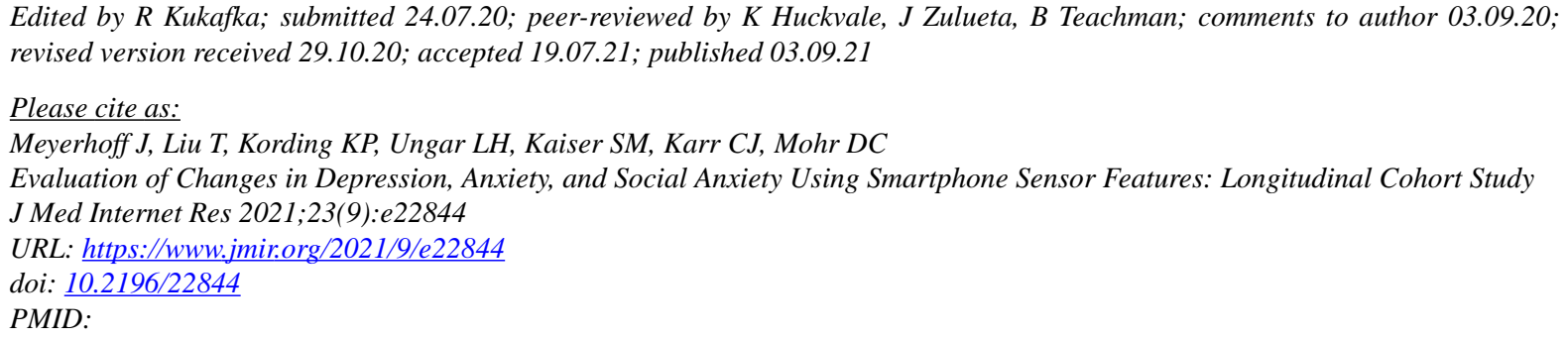

(CJonah Meyerhoff, Tony Liu, Konrad P Kording, Lyle H Ungar, Susan M Kaiser, Chris J Karr, David C Mohr. Originally published in the Journal of Medical Internet Research (https://www.jmir.org), 03.09.2021. This is an open-access article distributed under the terms of the Creative Commons Attribution License (https://creativecommons.org/licenses/by/4.0/), which permits unrestricted use, distribution, and reproduction in any medium, provided the original work, first published in the Journal of Medical Internet Research, is properly cited. The complete bibliographic information, a link to the original publication on https://www.jmir.org/, as well as this copyright and license information must be included. 